\title{
Ergonomics, user comfort, and performance in standard and robot-assisted laparoscopic surgery
}

\author{
R. H. van der Schatte Olivier · C. D. P. van't Hullenaar • \\ J. P. Ruurda · I. A. M. J. Broeders
}

Received: 7 February 2008/ Accepted: 13 August 2008/Published online: 15 October 2008

(c) The Author(s) 2008. This article is published with open access at Springerlink.com

\begin{abstract}
Background Robot-assisted surgical systems have been introduced to improve the outcome of minimally invasive surgery. These systems also have the potential to improve ergonomics for the surgeon during endoscopic surgery. This study aimed to compare the user's mental and physical comfort in performing standard laparoscopic and robot-assisted techniques. Surgical performance also was analyzed.

Methods In this study, 16 surgically inexperienced participants performed three tasks using both a robotic system and standard laparoscopic instrumentation. Distress was measured using questionnaires and an ambulatory monitoring system. Surgical performance was analyzed with time-action analysis.

Results The physiologic parameters $(p=0.000)$, the questionnaires $(p=0.000)$, and the time-action analysis ( $p=0.001)$ favored the robot-assisted group in terms of lower stress load and an increase in work efficiency.

Conclusion In this experimental setup, the use of a robotassisted surgical system was of value in both cognitive and
\end{abstract}

R. H. van der Schatte Olivier - C. D. P. van't Hullenaar .

J. P. Ruurda

Department of Surgery, University Medical Center, Utrecht,

The Netherlands

C. D. P. van't Hullenaar

e-mail: cas_vthullenaar@yahoo.com

C. D. P. van't Hullenaar · I. A. M. J. Broeders ( $\square)$

Department of Surgery, Meander Medical Center,

P.O. Box 1502, 3800 BM Amersfoort, The Netherlands

e-mail: iamj.broeders@meandermc.nl physical stress reduction. Robotic assistance also demonstrated improvement in performance.

Keywords Ergonomics - Laparoscopy ·

Minimally invasive surgery $\cdot$ Robotics $\cdot$ Stress

$\begin{array}{ll}\text { Abbreviations } \\ \text { AMS } & \text { Ambulatory monitoring system } \\ \text { HRA } & \text { Heart rate average } \\ \text { MSSD } & \begin{array}{l}\text { Mean square of successive differences between } \\ \text { consecutive heartbeats }\end{array} \\ \text { PEP } & \begin{array}{l}\text { Pre-ejection period } \\ \text { RA }\end{array} \\ \text { Robot assisted } \\ \text { RSA } & \text { Respiratory sinus arrhythmia } \\ \text { SL } & \text { Standard laparoscopic }\end{array}$

Laparoscopic surgery offers distinct advantages to patients over open surgery such as shorter hospitalization, reduced postoperative pain, and better cosmetic results [21, 29, 31]. On the other hand, laparoscopic surgery is technically and physically demanding for the surgeon due to limitations in manipulation and visualization as well as physical discomfort [2, 4, 5, 10, 14, 16, 23-25].

Robot-assisted surgical systems were developed to overcome many of the surgical disadvantages associated with laparoscopic surgery. Manipulation of the instruments is improved by increased range of motion for the instruments, correction of the inverted instrument response, and elimination of human tremors [1, 8, 20, 27]. Furthermore, vision of the operative field is improved by conversion of a two dimensional (2D) image to a 3D view. Additional advantages are a completely stable image and personal camera control. The physical 
discomfort of laparoscopic instrument handling is potentially reduced by restoration of the eye-hand-target working axis and physical support of the console [3, 18, $24,26]$. These advantages may reduce both the physical and mental workload, thus possibly improving both surgical performance and work satisfaction.

This experiment aimed to assess whether robot-assisted surgery benefits the surgeon by reducing mental and physical discomfort in comparison with standard laparoscopy. Additionally, task performance was evaluated to assess whether robot assistance can benefit surgical procedures [22].

\section{Methods}

In this study, 16 medical students ( 8 women and 8 men) each performed three tasks. These tasks were executed in a pelvic trainer in random order using both standard laparoscopic instruments (standard group) and the da Vinci robot-assisted surgical system (Intuitive Surgical, Sunnyvale, CA, USA) (robot-assisted group).

The participants were instructed in the tasks and techniques. To become familiar with the setup of the exercise, both the standard group and the robot-assisted group were allowed to practice each task for $2.5 \mathrm{~min}$. All the tasks were executed for $5 \mathrm{~min}$ with the objective to achieve as many repetitions as possible. The participants had a 15-min break after finishing all three tasks, during which they were asked to complete the questionnaires. After the break, the participant performed all three tasks again, but now in the other group. The tasks were rope passing, needle capping and bead dropping. All tasks are described here below. At the end of this second session, the participants completed the questionnaires again. To encourage maximal performance, the most accomplished participant was given a bonus.

In the standard laparoscopic group, table height was adjusted to the individual's preference. A horizontal instrument angulation less than $45^{\circ}$ and a vertical angulation less than $15^{\circ}$ [4] were always preserved. The monitor trolley was placed in line with the camera at a distance of $1.5 \mathrm{~m}$ from the pelvic trainer. The participants performed all the tasks using two dissection forcipes (B. Braun, Tuttlingen, Germany).

The robot-assisted group worked at the console, from which they controlled a three-armed da Vinci robot. Before onset of the tasks, each participant adjusted the console to his or her personal preference with regard to height of the chair, display clarity, optimal stereoscopic view, and comfortable armrest position. Both groups performed the same three tasks, and both groups performed all tasks with the same triangulation in the port placement setup.
Surgical tasks [11]

The following surgical tasks were performed.

\section{Rope passing}

A $25-\mathrm{cm}$-long rope (diameter, $0.3 \mathrm{~cm}$ ) was passed as many times as possible. The rope was grasped alternately with the left and right instruments at the 11 marked grasping points. During this exercise, the participants were instructed to keep the rope above the floor of the training box.

\section{Needle capping}

A needle and a cap were grasped and lifted, after which the needle was capped repeatedly above the floor of the training box.

\section{Bead dropping}

Every participant was asked to pick up a plastic bead from a starting position, transfer it crosswise, and drop it into a receptacle. After the bead was dropped into the receptacle, it had to be picked up and transferred to another receptacle using the contralateral laparoscopic instrument.

Physiologic measurements of mental discomfort

An ambulatory monitoring system was used for registration of physical parameters. It could be worn beneath clothing, allowing the subject complete freedom of movement [12, 28, 32]. Three physiologic stress parameters were recorded with this device.

The first parameter was the mean square of successive differences between consecutive heartbeats (MSSD). This parameter reflects the beat-to-beat variability of the heart rate and is tightly linked to respiratory sinus arrhythmia (RSA). Changes in RSA can display changes in vagal activity [6]. If vagal activity decreases RSA, MSSD also will be reduced because fewer oscillatory changes in heart rate occur. An increase in stress will therefore lead to a decrease in MSSD. In conclusion, high MSSD values reflect low stress levels.

The second parameter recorded was the preejection period (PEP: time of isovolumetric contraction). The PEP is the interval between the onset of ventricular depolarization and the opening of the semilunar valves [19]. Changes in PEP correspond to changes in B-adrenergic inotropic drive to the left ventricle. This correlation was shown by laboratory studies in which $\beta$-adrenergic tone was manipulated by epinephrine infusion $[15,30]$, adrenoceptor blockade [13], exercise [14, 17], and emotional stress [7, 18]. 
The third parameter was the average heart rate (HRA) of the participants recorded by the ambulatory monitoring system.

All three parameters (MSSD, PEP, HRA) were constantly recorded during both the laparoscopic tasks and the robot-assisted tasks. The data were automatically divided by the ambulatory monitoring system device into 30-s periods. The average of these 30-s recordings was compared with a paired sample $t$-test using SPSS software, version 13.0 for Windows (SPSS Inc., Chicago, IL, USA).

\section{Questionnaires}

The participants completed two questionnaires. To score the level of stress, all the participants completed the Subjective Mental Effort Questionnaire (SMEQ) and the Local Experienced Discomfort (LED) scale. The SMEQ is a cognitive workload questionnaire with a scale of 0 to 150 points (Appendix 1). It is designed so that individuals can rate the amount of effort invested during a task. Nine scale markers with verbal statements ranging from "no effort at all" to "exceptional amount of effort" are displayed in the SMEQ diagram. The choice of statements and their scale locations are empirically derived. The SMEQ, used as a tool in various laboratory and field studies, is categorized as a cognitive test [33].

The LED allowed participants to express their physical discomfort during performance of all the tasks [9]. On a scale of 0 to 10 points, the participants were asked to identify their physical discomfort at several locations of the upper body (Appendix 2). Statistical analysis of both questionnaires was performed using a Mann-Whitney $U$ test.

\section{Task performance}

All the procedures were recorded with a super-VHS recorder. Using these recordings, failures and successes of executed repetitions were applied to compare task performance between the two groups. The judgment for success or failure of a given task was based on consensus of the researchers.

\section{Results}

Physiologic measurements

Table 1 presents the physiologic measurements. The mean MSSD in the robot-assisted group was $31.7 \mathrm{~ms}$. This was significantly higher than in the standard laparoscopic group, which had a value of $22.3 \mathrm{~ms}(t=-4.0 ; p=0.01)$.

The results of the PEP also were in favor of the robotassisted group. A mean PEP value of $131.6 \mathrm{~ms}$ was recorded in the robot-assisted group, compared with a mean PEP value of $126.0 \mathrm{~ms}$ in the standard group $(t=3.3 ; p=0.004)$.

The average HRA was significantly higher in the standard laparoscopy group. A value of 90.5 beats/min was recorded in the standard laparoscopy group compared with a value of 79.9 beats $/ \min (t=5.1 ; p=0.0001)$ calculated for the robot-assisted group.

\section{Questionnaires}

The SMEQ questionnaire showed a median physical effort in the robot-assisted group of 30 points (range, 10-70), whereas a median of 80 points (range, 50-100) was reported for the standard laparoscopic group $(p=0.001)$. The LED score for the physical discomfort experienced was 7.4 points (range, 0-25) in the standard laparoscopic group compared with 1.3 points (range, $0-9$ ) in the robotassisted group $(p=0.001)$. The median number of physical complaints was 1 (range, $0-8$ ) in the standard group and 0 (range, $0-8)$ in the robot-assisted group $(p=0.003)$.

Time-action analysis

In each of the executed tasks of the experiment, the participants performed significantly more repetitions with robot assistance (Table 2). Additionally, fewer failures in task performance were recorded in the robot-assisted group than in the laparoscopic group. This discrepancy was statistically significant $(p<0.001)$.

Table 1 Physiologic measurements of the ambulatory monitoring system ${ }^{\mathrm{a}}$

\begin{tabular}{|c|c|c|c|c|}
\hline & Standard laparoscopy group & Robot assisted group & $t$-value & $p$-value \\
\hline MSSD (ms) & $22.3(8.0-73.4)$ & $31.7(15.0-53.4)$ & -4.0 & 0.01 \\
\hline PEP (ms) & $126.0(106.5-142.4)$ & $131.6(119.0-146.2)$ & 3.3 & 0.004 \\
\hline HRA (beats/min) & $90.5(73.8-109.4)$ & $79.9(66.9-98.0)$ & 5.1 & 0.0001 \\
\hline
\end{tabular}

$M S S D$ mean square of successive differences between consecutive heartbeats, $P E P$ between consecutive heartbeats, $H R A$ average heart rate

${ }^{a}$ Data are presented as mean value (range) 
Table 2 Results of the action analysis ${ }^{\mathrm{a}}$

\begin{tabular}{|c|c|c|c|c|c|}
\hline & \multicolumn{2}{|c|}{ Standard lap } & \multicolumn{2}{|c|}{ Robot assisted } & \multirow[t]{2}{*}{$p$-value } \\
\hline & Median & Range & Median & Range & \\
\hline Rope passing & 3.5 & $1.0-7.5$ & 8.3 & $5.5-14.0$ & 0.000 \\
\hline Failure to pass the rope & 5.0 & $1.0-4.0$ & 1.5 & $0.0-5.0$ & 0.000 \\
\hline Needle capping & 1.0 & $0.0-6.0$ & 5.0 & $2.0-10.0$ & 0.000 \\
\hline \multicolumn{6}{|l|}{ Failure to cap the needle } \\
\hline Missing the cap & 13.0 & $2.0-25.0$ & 0.5 & $0.0-9.0$ & 0.000 \\
\hline Dropping the needle & 6.5 & $4.0-14.0$ & 2.0 & $1.0-9.0$ & 0.001 \\
\hline Bead drop & 22.0 & $3.0-45.0$ & 48.0 & $28.0-69.0$ & 0.000 \\
\hline Failure to drop the bead into the receptacle & 3.0 & $1.0-7.0$ & 0.0 & $0.0-5.0$ & 0.001 \\
\hline
\end{tabular}

${ }^{a}$ Number of repetitions is expressed as median and range. Mann-Whitney $U$ test used to calculate $p$ value

\section{Discussion}

This study aimed primarily to assess whether robot-assisted surgery can benefit the surgeon in terms of stress reduction and physical comfort. In general, stress can be defined as a specific response by the body to a stimulus that disturbs or interferes with the normal physiologic equilibrium. This physical, mental, or emotional tension of an individual decreases the feeling of being in control of a given situation.

Surgeons control the surgical environment usually by means of mental and physical skills, in cooperation with operating room colleagues and the available equipment. Improving either one of these aspects can increase situational control. In this experiment, the focus was placed on the equipment.

Robot-assisted surgical systems are designed to facilitate manipulation of surgical instruments by increasing freedom of movement and introducing stereoscopic vision. Additionally, physical workload is reduced as the surgeon operates from a comfortable console instead of standing next to the operating table [14]. Ergonomics and user comfort in robot-assisted laparoscopic surgery have not gained much scientific interest but probably are underestimated in an era when more endoscopic procedures are being performed every year, both in basic and complex surgery. A recent study suggests a possible decrease in mental stress with the implementation of robotic techniques, but statistical significance to support this finding has not been established [5].

Our study confirmed that robotic assistance can reduce physical stress significantly. The objective recordings of the ambulatory monitoring system device (MSSD, PEP, HRA) demonstrated robot-assisted surgery to be less stressful than standard laparoscopy. Besides these physical parameters, the mental questionnaires also displayed a significant decrease in mental and physical effort when laparoscopic tasks were performed with the assistance of a robot.

The questionnaires demonstrate that robotic assistance reduced the cognitive workload $(p<0.0001)$, the amount of physical discomfort $(p=0.001)$, and the total number of complaints $(p=0.003)$. The performance of all the tasks in the robotic group was significantly better, with more repetitions and fewer failures.

These results all are highly suggestive of stress reduction. However, some critical remarks must be made. First, physical and mental stress in simulated, experimental conditions is not directly comparable with stress in actual surgical situations. In actual procedures, with teamwork also playing a substantial role, the working environment can be more complex and demanding. The seated position of the participants in the robot-assisted group must be taken into account as well.

Second, with a recording time of 15 min (excluding 7.5 min of practice time), the "operating time" was relatively short. It is likely that more physical discomfort and fatigue arises during actual procedures. This can influence our results [14].

Third, the research group consisted of inexperienced participants without specific surgical skills. Because the effect of surgical skills on the reduction of stress is not known, it is hard to determine whether our research group is comparable with a population of laparoscopic surgeons.

To a certain extent, the aforementioned factors may have affected our results. However, our results clearly indicate that improvement in manipulation and the introduction of a stereoscopic view in a comfortable and ergonomic workspace can contribute to an increased feeling of situational control and a decreased level of physical workload. In conclusion, the implementation of a robotic system for the execution of laparoscopic tasks in an experimental setup enhances performance and reduces cognitive stress levels as well as physical discomfort. 


\section{Appendix 1}

Subjective Mental Effort Questionnaire (SMEQ)

Questionnaire [33]

SMEQ Questionnaire [33]

This graphic displays the amount of effort it took you to execute the task you have been working on.

Please score the amount of effort by marking one of the anchors on the vertical line here below.

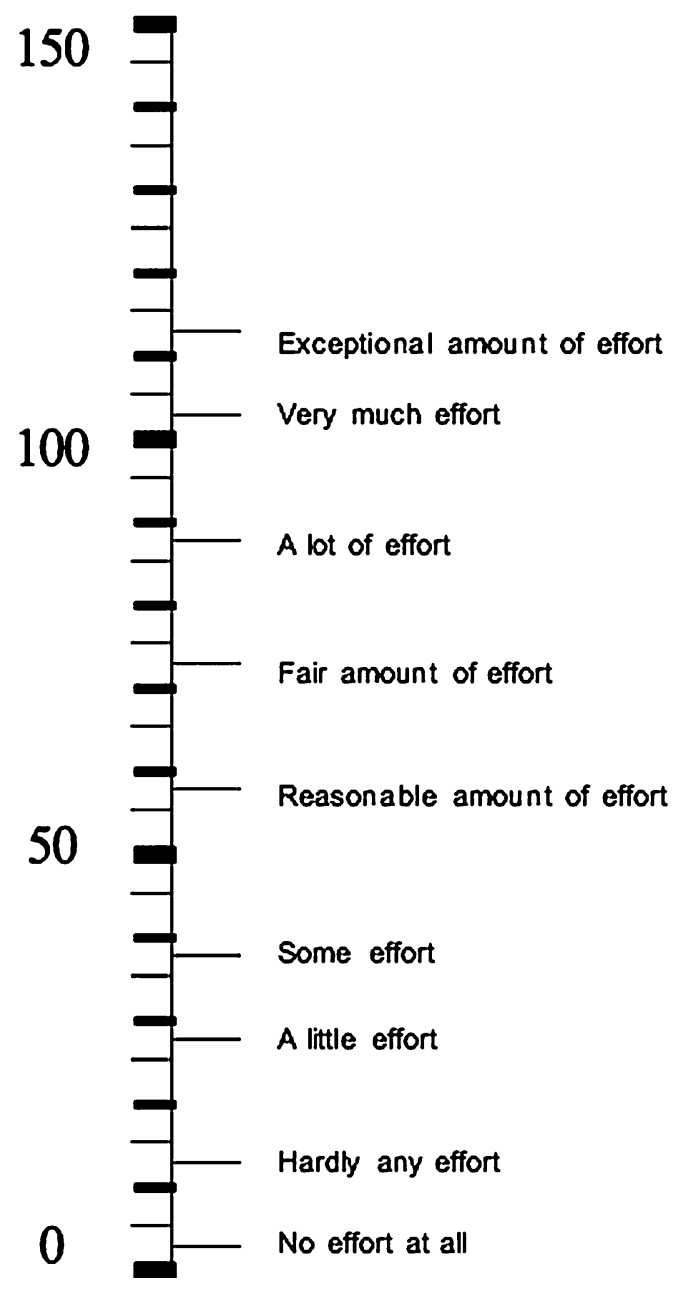




\section{Appendix 2}

Local Experienced Discomfort (LED) Scale [9]



For each of the body parts indicated by the letters in the picture, please fill in a score as presented here below.

$\begin{array}{ll} & \text { Maximum } \\ 10 & \text { Extreme amount of complaints } \\ 9 & \\ 8 & \\ 7 & \text { A lot of complaints } \\ 6 & \\ 5 & \\ 4 & \text { Quite a lot complaints } \\ 3 & \\ 2 & \text { Some complaints } \\ 1 & \\ 1 / 2 & \text { Hardly any complaints } \\ 0 & \text { No complaints at all } \\ & \text { Minimum }\end{array}$

Open Access This article is distributed under the terms of the Creative Commons Attribution Noncommercial License which permits any noncommercial use, distribution, and reproduction in any medium, provided the original author(s) and source are credited.

\section{References}

1. Ballantyne GH, Hourmont K, Wasielewski A (2003) Telerobotic laparoscopic repair of incisional ventral hernias using intraperitoneal prosthetic mesh. JSLS 7:7-14

2. Berggren U, Gordh T, Grama D, Haglund U, Rastad J, Arvidsson D (1994) Laparoscopic versus open cholecystectomie:

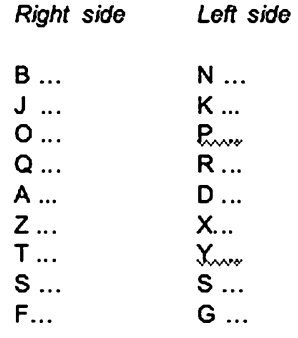

hospitalization, sick leave, analgesia, and trauma responses. Br J Surg 81:1362-1365

3. Berguer R (1998) Surgical technology and the ergonomics of laparoscopic instruments. Surg Endosc 12:458-462

4. Berguer R, Forkey DL, Smith WD (2001) The effect of laparoscopic instrument working angle on surgeons' upper extremity workload. Surg Endosc 15:1027-1029

5. Berguer R, Smith W (2006) An ergonomic comparison of robotic and laparoscopic technique: the influence of surgeon experience and task complexity. J Surg Res 134:87-92

6. Berne MR, Levy MN, Stanton BA, Koeppen BM (2006) Berne \& Levy principles of physiology, 4th edn. Elsevier Mosby, Philadelphia, PA, pp 383-385

7. Berntson GG, Cacioppo JT, Binkley PF, Uchino BN, Quigley KS, Fieldstone A (1994) Autonomic cardiac control: III. 
Psychological stress and cardiac response in autonomic space as revealed by pharmacological blockades. Psychophysiology 31: 599-608

8. Bowersox JC, Shah A, Jensen J, Hill J, Cordts PR, Green PS (1996) Vascular applications of telepresence surgery: initial feasibility studies in swine. J Vasc Surg 23:281-287

9. Corlett EN, Bishop RP (1976) A technique for measuring postural discomfort. Ergonomics 9:175-182

10. Emam TA, Hanna G, Cuschieri A (2002) Ergonomic principles of task alignment, visual display, and direction of execution of laparoscopic bowel suturing. Surg Endosc 16:267-271

11. Garcia-Ruiz A, Gagner M, Miller JH, Steiner CP, Hahn JF (1998) Manual vs robotically assisted laparoscopic surgery in the performance of basic manipulation and suturing tasks. Arch Surg 133:957-961

12. de Geus EJC, Willemsen GHM, Klaver CHAM, van Doornen LJP (1995) Ambulatory measurement of respiratory sinus arrythmia and respiration rate. Biol Psychol 41:205-227

13. Harris WS, Schoenfeld CD, Weissler AM (1967) Effects of adrenergic receptor activation and blockade on the systolic preejection period, heart rate, and arterial pressure in man. J Clin Invest 46:1704-1714

14. Johnston WKIII, Hollenbeck BK, Wolf JS Jr (2005) Comparison of neuromuscular injuries to the surgeon during hand-assisted and standard laparoscopic urologic surgery. J Endourol 19:377-381

15. Krzeminski K, Kruk B, Nazar K, Ziemba AW, Cybulski G, Niewiadomski W (2000) Cardiovascular, metabolic, and plasma catecholamine responses to passive and active exercises. J Physiol Pharmacol 51:267-278

16. Majeed AW, Jacob G, Reed MW, Johnson AG (1993) Laparoscopist's thumb: an occupational hazard. Arch Surg 128:357

17. Mezzacappa ES, Kelsey RM, Katkin ES (1999) The effects of epinephrine administration on impedance cardiographic measures of cardiovascular function. Intern J Psychophysiol 31:189-196

18. Miyamoto Y, Higuchi J, Abe Y, Hiura T, Nakazono Y, Mikami T (1983) Dynamics of cardiac output and systolic time intervals in supine and upright exercise. J Appl Physiol 55:1674-1681

19. Newlin DB, Levenson RW (1979) Pre-ejection period: measuring B-adrenergic influences upon the heart. Psychophysiology $16: 546-553$
20. Nifong LW, Chu VF, Bailey BM, Maziarz DM, Sorrell VL, Holbert D, Chitwood WR Jr (2003) Robotic mitral valve repair: experience with the da Vinci system. Ann Thorac Surg 75:438-442

21. Nilsson G, Larsson S, Johnsson F (2000) Randomized clinical trial of laparoscopic versus open fundoplication: blind evaluation of recovery and discharge period. Br J Surg 87:873-878

22. Nio D, Bemelman WA, den Boer KT, Dunker MS, Gouma DJ, van Gulik TM (2002) Efficiency of manual vs robotical (Zeus) assisted laparoscopic surgery in the performance of standardized tasks. Surg Endosc 16:412-415

23. Patkin M, Isabel L (1995) Ergonomics, engineering and surgery of endosurgical dissection. J R Coll Surg Edinb 40(2):120-132

24. Prasad SM, Maniar HS, Soper NJ, Damiano RJ Jr, Klingensmith ME (2002) The effect of robotic assistance on learning curves for basic laparoscopic skills. Am J Surg 183:702-707

25. Renda A, Vallancien G (2003) Principles and advantages of robotics in urologic surgery. Curr Urol Rep 4:114-118

26. Ruurda JP, van Vroonhoven ThMJ, Broeders IAMJ (2002) Robot-assisted surgical systems: a new era in laparoscopic surgery. Ann R Coll Surg Engl 84:223-226

27. Ruurda JP, Broeders IA (2003) Robot-assisted laparoscopic intestinal anastomosis. Surg Endosc 17(2):236-241

28. Sherwood A, Allen MT, Fahrenberg J, Kelsey RM, Lovallo WR, van Doornen LJP (1990) Methodological guidelines for impedance cardiography. Psychophysiology 27:1-23

29. Stiff G, Rhodes M, Kelly A, Telford K, Armstrong CP, Rees BI (1994) Long-term pain: less common after laparoscopic than open cholecystectomy. Br J Surg 81:1368-1370

30. Svedenhag J, Martinsson A, Ekblom B, Hjemdahl P (1986) Altered cardiovascular responsiveness to adrenaline in endurance-trained subjects. Acta Physiol Scand 126:539-550

31. Vanek VW, Rhodes R, Dallis DJ (1995) Results of laparoscopic versus open cholecystectomie in a community hospital. South Med J 88:555-566

32. Willemsen GHM, de Geus EJC, Klaver CHAM, van Doornen LJP, Carroll D (1996) Ambulatory monitoring of the impedance cardiogram. Psychophysiology 33:184-193

33. Zijlstra FRH (1993) Efficiency in work behaviour: a design approach for modern tools. $\mathrm{PhD}$ thesis, Delft University of Technology. Delft, The Netherlands, Delft University Press 